Disponível em

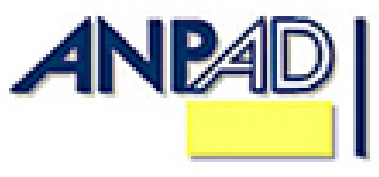

http://www.anpad.org.br/rac

RAC, Rio de Janeiro, v. 16, n. 4, art. 6, pp. 608-627, Jul./Ago. 2012

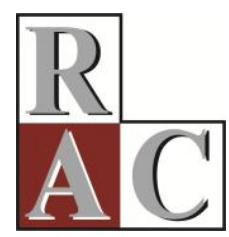

\title{
Impactos do Índice Dow Jones, Commodities e Câmbio sobre o Ibovespa: uma Análise do Efeito Contágio ${ }^{(1)}$
}

Effects of the Dow Jones Index, Commodities and Exchange Rate on Ibovespa: an Analysis of Contagion Effects

Pedro Raffy Vartanian* E-mail: pedro.vartanian@mackenzie.br Universidade Presbiteriana Mackenzie. São Paulo, SP, Brasil.

\footnotetext{
* Endereço: Pedro Raffy Vartanian

Universidade Presbiteriana Mackenzie, Rua da Consolação, 930, Consolação, São Paulo/SP, 01302-907.
}

Copyright (C) 2012 RAC. Todos os direitos, até mesmo de tradução, são reservados. É permitido citar parte de artigos sem autorização prévia, desde que seja identificada a fonte. 


\title{
Resumo
}

O objetivo da pesquisa é avaliar a existência do efeito contágio do índice Dow Jones, preços das commodities e taxa de câmbio sobre a trajetória do Ibovespa no período 1999-2010, além de verificar as relações de longo prazo entre as variáveis. O marco teórico baseia-senoefeito contágio, em que ocorre a propagação de perturbações no mercado de um país para outro, conforme abordado por Dornbusch, Park e Claessens (2000), Pericoli e Sbracia (2003) e Forbes e Rigobon (2002), além de estudos empíricos como os de Lamounier e Nogueira (2007), Tabak e Lima (2003), Grôppo (2006) e Pimenta (2004), entre outros, complementando com pesquisas dos impactos dos preços de commodities sobre o mercado acionário, conforme pode ser observado em Barr e Kantor (2002). Para tanto, foi aplicado teste de cointegração de acordo com procedimento sugerido por Johansen (1991), além de um modelo de vetores autorregressivos (VAR), proposto inicialmente por Sims (1980) e Sims (1986). Os resultados do teste de cointegração de Johansen não indicaram a existência de relações de longo prazo entre as variáveis. Em termos dos efeitos de curto prazo, as funções de resposta a impulso mostraram que o índice de ações brasileiro reage positivamente aos choques nos preços das commodities e ao índice Dow Jones, além de demonstrar uma reação positiva à depreciação cambial, o que sugere a presença do efeito contágio.

Palavras-chave: integração de mercados financeiros; mercado acionário; modelo VAR; efeito contágio.

\begin{abstract}
The purpose of this research is to evaluate the existence of contagion effects of the Dow Jones index, commodity prices and exchange rate on the trajectory of the Bovespa index in the period 1999-2010, and analyze the longterm relationships between the variables. The theoretical framework is based on contagion effect, which occurs in the spread of market disturbances from one country to another country as discussed by Dornbusch, Park and Claessens (2000), Pericoli and Sbracia (2003), and Forbes and Rigobon (2002), as well as in empirical studies such as Lamounier and Nogueira (2007), Tabak e Lima (2003), Groppo (2006) and Pimenta (2004), among others, complete with research of the impact on commodity prices in the stock market, as can be seen in Barr and Kantor (2002). For this purpose, a cointegration test from the procedure suggested by Johansen (1991) was applied, and a vector autoregression model (VAR), initially proposed by Sims (1980) and Sims (1986), with the causality/exogeneity Granger test, was utilized. The results of Johansen cointegration test did not indicate the existence of long-term relationships between the variables. As to short-term effects, the impulse response functions showed that the Brazilian stock market index reacts positively to shocks in commodity prices and the U.S. stock market, and it demonstrates a positive reaction to currency depreciation, which suggests presence of the contagion effect.
\end{abstract}

Key words: financial market integration; stock market; VAR model; contagion effect. 


\section{Introdução}

A ampliação das conexões entre os mercados financeiros internacionais tornou-se evidente, em 2007, com os primeiros efeitos da crise do mercado de crédito imobiliário norte-americano sobre o mercado internacional de capitais. Naquele mesmo ano, após o índice Dow Jones atingir a máxima histórica em número de pontos, teve início um processo de reversão que perdurou durante todo o ano seguinte e afetou outros mercados, incluindo o mercado acionário brasileiro. Alguns meses depois do processo de reversão da bolsa estadunidense, os preços das ações das empresas brasileiras seguiram o mesmo padrão, com um processo de reversão de tendência, após alcançar a máxima histórica em maio de 2008. De forma quase que concomitante, os preços das principais commodities apresentaram comportamento similar, com uma súbita reversão de tendência inédita, do ponto de vista histórico, tanto em termos de velocidade quanto de amplitude.

A crise estadunidense resultou claramente em mais um episódio de efeito contágio. $\mathrm{O}$ efeito contágio foi amplamente discutido na literatura, principalmente a partir da segunda metade da década de 1990, com a ocorrência de crises em mercados emergentes (México, Rússia, Ásia e Brasil). Não há uma única definição para o efeito contágio, ainda que, geralmente, seja compreendido como a propagação de perturbações no mercado cambial ou acionário de um país para outro, conforme abordado por Dornbusch, Park e Claessens (2000). Esta definição aproxima-se da utilizada por Forbes e Rigobon (2002), mas difere ligeiramente do estudo de Pericoli e Sbracia (2003), cuja delimitação apresentada constitui uma entre as cinco principais definições de contágio.

Nesse sentido, abordagens sobre conexões entre mercados financeiros, incluindo o comportamento das ações brasileiras, aparecem em estudos que tiveram o objetivo de avaliar a possibilidade de diversificação de risco em mercados internacionais e de avaliação do efeito contágio. A utilização de modelos de vetores autorregressivos (VAR), como no estudo de Pimenta (2004), e técnicas de avaliação de cointegração, como no trabalho de Tabak e Lima (2003), constituem metodologias recorrentes na agenda de pesquisa. É evidente, no entanto, que o comportamento do mercado acionário de um país também se relaciona com variáveis macroeconômicas de natureza interna, como demonstrou Grôppo (2006), que destacou a taxa de câmbio como uma das variáveis que mais se relacionou com o comportamento das ações no período de 1995 a 2005. O ingresso de investimento estrangeiro, ocasionado pela estabilidade da moeda nacional, associado ao aumento da integração dos mercados financeiros internacionais, vem justificando a relação entre os mercados cambial e acionário.

Considerando, portanto, que o regime cambial brasileiro, em vigor a partir de 1999, é classificado como regime de flutuação pura, de acordo com o Fundo Monetário Internacional (FMI, 2008), o objetivo do presente estudo é avaliar se a trajetória do Ibovespa no período 1999-2010 se relacionou com o comportamento das ações norte-americanas, representadas pelo índice Dow Jones e/ou com os preços das commodities no mercado internacional, cuja variável representativa, neste estudo, é o índice de commodities Reuters-Jefferies, além, evidentemente, da taxa de câmbio. Para tanto, foi aplicado teste de cointegração entre o índice e as variáveis selecionadas, além da estimativa por meio de um modelo de vetores autorregressivos (VAR), com teste de causalidade/exogeneidade de Granger objetivando identificar o efeito contágio. A hipótese da pesquisa é que o Ibovespa foi influenciado pelo desempenho dos preços de commodities e pelo comportamento da bolsa americana no período analisado e que há um comportamento de cointegração entre as variáveis selecionadas.

A pesquisa justifica-se pela abordagem do comportamento da trajetória do Ibovespa exclusivamente sob um regime de câmbio flutuante, relacionando-o com o preço das commodities e com o índice Dow Jones, além das variações na taxa de câmbio, o que consubstancia o estudo ao período 1999-2010. Vale destacar que, sob um regime de flutuação cambial, a dinâmica dos preços das ações é distinta se comparada a um regime cambial rígido, como o regime de bandas cambiais utilizado na economia brasileira, no período de 1995 até início de 1999. Tem-se, neste contexto, um canal adicional de contágio da taxa de câmbio para o mercado acionário. 
Adicionalmente, a necessidade de identificar possíveis relações entre os preços das commodities e o índice de ações brasileiro deriva da forte concentração do Ibovespa em empresas de setores relacionados à exploração, produção e/ou comercialização de matérias-primas em âmbitos nacional e internacional, além da possibilidade de avaliar o efeito contágio com a consequente viabilidade de diversificação entre aplicações na bolsa brasileira e em fundos de commodities, tendo em vista que transações envolvendo commodities aumentaram ao longo da década de 2000, com destaque para a criação de fundos de investimento específicos para commodities. Destaca-se, ainda, que a literatura acerca dos impactos e contágio de preços de commodities sobre o mercado acionário é limitada, tanto para o caso brasileiro quanto para outros países que possuem índices de ações fortemente concentrado sem empresas de commodities, o que justifica a importância da pesquisa.

Os resultados encontrados indicam que há um efeito contágio positivo do preço das commodities sobre o Ibovespa, além de um efeito, também positivo, do índice Dow Jones sobre a bolsa brasileira. Adicionalmente, constatou-se inexistência de cointegração entre as variáveis, o que permite a diversificação de investimentos entre ações do Brasil, dos EUA e fundos de commodities ao longo prazo.

Para tanto, este artigo apresenta, na segunda seção, aspectos teóricos sobre o efeito contágio e uma revisão de trabalhos empíricos, incluindo pesquisas sobre impactos de preços de commodities em outros índices. A terceira seção discorre sobre a metodologia e os dados utilizados na pesquisa, enquanto a quarta seção apresenta e discute os resultados. Finalmente, na quinta seção, são apresentadas as considerações finais.

\section{Referencial Teórico}

O marco teórico do trabalho tem origem no chamado efeito contágio, conforme mencionado anteriormente. Desse modo, inicialmente, a seção contempla a definição do efeito contágio. Posteriormente, serão apresentados resultados de trabalhos empíricos que avaliaram a relação entre distintos mercados, com ênfase para os efeitos sobre o mercado brasileiro. Vale destacar ainda que a literatura contempla, de forma recorrente, conexões e análise de contágio entre mercados acionários, mas a abordagem da relação entre preços de commodities e mercado acionário é quase inexistente.

A definição de contágio recorrente na literatura pode ser vista em Dornbusch et al. (2000). Segundo os autores, contágio refere-se à propagação de perturbações nos mercados de um país para outro, em um processo de comovimento nas taxas de câmbio, ações, títulos soberanos e fluxos de capital. Essa definição aproxima-se da utilizada por Forbes e Rigobon (2002). No entanto, Forbes e Rigobon (2002) diferenciam o efeito contágio da interdependência. A interdependência ocorre quando o comovimento não aumenta significativamente após um choque, enquanto o contágio mostra um aumento no comovimento diante de um choque qualquer. Adicionalmente, Pericoli e Sbracia (2003) apresentam uma revisão da literatura atinente ao tema destacando as cinco principais definições de contágio. Uma delas alude especificamente ao mercado cambial quando define o contágio como o aumento da probabilidade de crise em um determinado país, com base em uma crise que esteja ocorrendo em outro país. Outra abordagem contempla o mercado financeiro, a qual define o contágio como um processo em que a volatilidade de um país em crise provoca um transbordamento (spillover) para os mercados financeiros de outros países. Há ainda outra definição relacionada aos mercados financeiros em que o contágio ocorre quando os comovimentos não são explicados pelos fundamentos econômicos.

Sob a ótica de pesquisas empíricas, é inequívoca a afirmação de que a literatura já apresenta inúmeras abordagens com as relações entre o Ibovespa e o Dow Jones, frequentemente, por meio da utilização da teoria de cointegração de Johansen (1991) ou por intermédio dos modelos VAR, originalmente propostos por Sims (1980). O avanço nas pesquisas resultou, ainda, em análises efetuadas a partir de modelos de vetores autorregressivos com a inclusão de vetores de cointegração, 
no que se convencionou chamar de modelo VEC (vector errorcorrection). Já a análise dos determinantes da trajetória do Ibovespa, exclusivamente sob um regime de câmbio flutuante, é menos recorrente, principalmente, em função de análises de cointegração e de causalidade exigirem amplitude temporal indisponível no período de realização das pesquisas.

Estudos iniciais pertinentes ao processo de integração de mercados financeiros, como o de Leal e Costa (1998), tiveram o objetivo de avaliar a integração das bolsas brasileira e argentina após a abertura financeira no início da década de 1990. Nesse sentido, a pesquisa identificou indícios de integração do mercado acionário brasileiro com o americano já a partir de 1993. Segundo os autores, que avaliaram também a integração do mercado argentino com o norte-americano, a estabilização das economias dos dois países, a abertura dos mercados financeiros e a emissão de títulos no exterior favoreceram o estreitamento das relações entre as bolsas de valores.

Em contrapartida, Sanvicente (1998) refutou a hipótese de cointegração entre o mercado acionário brasileiro e estadunidense, contemplando o período de janeiro de 1986 a dezembro de 1997 , com a utilização de dados diários do Ibovespa e do índice Dow Jones. A análise permitiu concluir que há possibilidade de diversificação entre os mercados, tendo em vista que, ao longo prazo, não há sinais de comportamento convergente, o que permite a diversificação por meio de aplicações nas duas bolsas.

Em um período mais recente e utilizando a teoria de cointegração, Tabak e Lima (2003) também não encontraram evidências de cointegração entre o mercado acionário brasileiro e outros mercados, como o argentino, mexicano e norte-americano. No período de análise, que teve início em janeiro de 1995 e se encerrou em março de 2001, a mudança do regime cambial e a consequente desvalorização do real provocaram uma forte queda no Ibovespa em pontos, o que pode ter indicado inexistência de cointegração entre o mercado acionário brasileiro e norte-americano. A estimativa realizada sem a inclusão de uma variável dummy, representando a mudança do regime cambial, pode ter contribuído para a não identificação de uma relação de cointegração. Contudo, na análise de contágio a curto prazo, aferida pelo teste de causalidade estatística, os autores encontraram evidências de que o mercado americano influencia o desempenho das ações nos mercados latino-americanos.Ainda assim, o estudo enfatiza a necessidade de desenvolver-se uma análise de quebras estruturais verificadas nos índices com o objetivo de detectar eventuais relações a longo prazo.

Em outro estudo, Caselani e Eid (2005) refutaram a tese de influência do mercado acionário norte-americano sobre o brasileiro, a partir de um modelo multivariado que evidenciou aspectos de retroalimentação da volatilidade e a relação com os retornos das ações. Os autores destacaram, ainda, que boas práticas de governança corporativa influenciaram o desempenho das ações no período selecionado para análise (1995-2003). Vale ressaltar que, pelo fato do período selecionado também ser caracterizado por uma mudança do regime cambial brasileiro, houve a inclusão de uma variável dummy na especificação do modelo.

Com outra estratégia de modelagem, Pereira, Dantas e Costa (2002) igualmente não identificaram relações de cointegração entre as bolsas americana e a brasileira. Em uma análise com dados semanais e índices expressos em dólares, a mudança do regime cambial não foi contemplada com variáveis dummies, o que pode ter prejudicado os testes de cointegração realizados. Apesar disso, os autores encontraram evidências de cointegração com as bolsas argentina e mexicana, com isso, sugerindo que, em momento de crise, por exemplo, os países são tratados da mesma maneira por investidores internacionais. Por sua vez, Grôppo, Amaral, Bertucci e Barros (2001), utilizando-se de testes de causalidade, identificaram interferências dos mercados norte-americano e argentino sobre o brasileiro, ainda que não tenha sido possível prever o comportamento das ações brasileiras com base na variação dos preços das ações norte-americanas, pois não se pôde identificar relações de cointegração entre os mercados.

Lamounier e Nogueira (2007) também identificaram efeitos a curto prazo entre mercados internacionais e o mercado brasileiro com testes de causalidade. A análise compreendeu dois períodos. O primeiro período analisado foi de setembro de 1995 a dezembro de 2002, e o segundo período 
analisado foi de janeiro de 2003 a agosto de 2005. A justificativa dos autores para a análise em dois momentos foi dada pela relativa estabilidade no segundo momento, o que permitiria uma comparação com o primeiro período, que foi caracterizado por crises internacionais. O estudo, todavia, utilizou os índices de bolsas em moedas locais, considerando que o risco cambial pode ser completamente eliminado por operações de hedge, o que não é recorrente tendo em vista os custos advindos da proteção da variação cambial. A abordagem concluiu que tanto o Ibovespa quanto as bolsas de países emergentes são influenciados, a curto prazo, pelas bolsas dos mercados norte-americano e londrino, ainda que a quebra estrutural do Ibovespa, em janeiro de 1999, possa ter produzido resultados viesados.

Em avaliação da interdependência entre os mercados, Pimenta (2004) avaliou a influência do Nasdaq sobre as bolsas da Argentina, Brasil, Chile e México, com a aplicação de um modelo VAR, decomposição da variância e funções de resposta a impulso. $O$ autor individuou efeitos de interdependência do mercado americano sobre as bolsas dos quatro países, analisando um período de doze anos que teve início em janeiro de 1992, o que também resultou em várias quebras estruturais na série do Ibovespa. Mesmo assim, o estudo detectou influência do Nasdaq sobre o Ibovespa, mas destacou que o país que apresentou relação mais estreita com os EUA foi o México.

Com o objetivo de avaliar a influência de variáveis de política monetária sobre o Ibovespa, Grôppo (2006) identificou a importância da taxa de câmbio no comportamento do Ibovespa em análise do período 1995-2005, com um modelo VEC que incluiu outras variáveis, como a oferta de moeda e as taxas de juros a curto e longo prazo. Como o autor contemplou dois regimes cambiais distintos na estimativa, foi inclusa uma variável dummy representando a mudança estrutural resultante da mudança do regime cambial de 1999. O procedimento tende a ser mais eficaz na estimativa e na confiabilidade dos resultados, pois, de acordo com Gamboa (2006), as quebras estruturais podem comprometer os resultados de testes econométricos, como a superaceitação de hipótese de raiz unitária e, no caso dos testes de cointegração, a superestimação do número de vetores de cointegração identificados. Mesmo assim, Gamboa (2006) destaca que não há um consenso na literatura quanto à melhor forma de se testar a cointegração em séries com quebras, o que se confirma na inexistência de rotinas padronizadas nos softwares e pacotes econométricos. Os resultados da análise de Grôppo (2006) evidenciaram que a maior sensibilidade do Ibovespa é justamente com a taxa de câmbio, entre as variáveis analisadas.

Já Nogueira e Lamounier (2008) buscaram identificar o efeito contágio entre bolsas de países emergentes com países desenvolvidos, em uma análise que contemplou o período de 1995 a 2005 . Os autores também supuseram possibilidade de eliminação do risco cambial e concluíram, por meio de testes de cointegração, que há possibilidade de diversificação no mercado internacional de capitais porque, mesmo com indícios de cointegração, os choques propagam-se de forma lenta, dessa maneira, permitindo aos investidores diversificar em períodos intermitentes. As relações de cointegração, nesse estudo, não permitiram apontar quais mercados são cointegrados, sendo que a bolsa brasileira também foi analisada sob dois períodos distintos em termos de regime cambial.

Como pode ser verificado, inúmeros autores abordaram relações de cointegração e/ou de causalidade do Ibovespa a partir do comportamento das ações do mercado estadunidense. Já a relação entre as bolsas de valores e os preços das commodities constitui-se como uma lacuna na literatura. Alguns estudos, como de Fifield, Power e Sinclair (2002), tiveram o objetivo de relacionar o desempenho das bolsas de valores de países emergentes com variáveis domésticas e internacionais, entre as quais, os preços das commodities. Assim, os autores incluíram a variável preço de commodities como uma das variáveis internacionais e não obtiveram um padrão em termos de efeitos sobre as bolsas de países emergentes. As bolsas da Índia e da China tiveram relação com fatores internos como produção interna, inflação e taxa de juros. Já as bolsas do México, Portugal e Singapura, entre outras, mostraram relação com fatores externos, tais como inflação internacional e produção mundial. Vale destacar que a bolsa brasileira não fez parte da amostra do estudo. Já Barr e Kantor (2002) relacionaram, por meio da metodologia de cointegração, o mercado acionário da África do Sul com os preços das commodities e com outros mercados acionários, encontrando evidências de uma relação positiva entre os preços das commodities e o das ações. 
De uma forma geral, observou-se que parte das pesquisas atentou para a importância do regime cambial sobre o comportamento do Ibovespa, de forma concomitante às estimativas que consideraram apenas o risco inerente às economias. Isso reforça a necessidade de uma análise de efeitos de cointegração e de causalidade para um período mais extenso, como o da presente pesquisa, em que o Ibovespa é analisado exclusivamente sob um regime de câmbio flutuante, com a inclusão de uma variável que representa os preços das commodities no mercado internacional.

\section{Metodologia e Dados}

O comportamento dinâmico das variáveis que se relacionam com o Ibovespa pode ser analisado por meio de um modelo temporal econométrico VAR, que tem como principal característica a capacidade de analisar as relações interligadas entre as variáveis escolhidas para compor o modelo. Além disso, o modelo pode captar o valor defasado (lags) das variáveis. O modelo VAR foi inicialmente proposto por Sims (1980), surgindo como alternativa aos modelos multiequacionais, em decorrência de os procedimentos utilizados nas estimativas terem sido considerados, pelo autor, como inapropriados $^{(2)}$. A questão da identificação nos modelos tradicionais, tratada com detalhes por Sims (1986), impõe uma série de restrições desnecessárias aos modelos econométricos e acarreta perdas de informações importantes.

A principal vantagem do modelo é a possibilidade de estimar diversas variáveis simultaneamente, evitando os problemas de identificação dos parâmetros em modelos multiequacionais. A modelagem VAR não foi absolvida de críticas ao longo dos últimos anos, o que não impediu sua utilização como um importante instrumento de análise e estimação de equações com duas ou mais variáveis ${ }^{(3)}$. Ademais, as críticas desencadearam pesquisas para que a estratégia de modelagem se desenvolvesse. Algumas propostas com relação às melhorias nas estimativas e confiabilidade estatística tornaram o modelo VAR de ampla utilização. Novos testes foram desenvolvidos e aprimorados, o que implicou aumento do poder preditivo e ampliação da confiabilidade nos coeficientes estimados e na análise de resultados.

A interpretação clássica do modelo VAR normalmente se dá por meio da função de resposta a impulso. As estimativas efetuadas por intermédio da função de resposta a impulso permitem avaliar adequadamente resultados de choques em qualquer uma das variáveis do sistema e têm sido a peça central na utilização de vetores autorregressivos. A partir do cálculo efetuado na estimativa, é possível visualizar graficamente o impacto de cada variável do sistema com relação às demais variáveis. A função de resposta a impulso representa, basicamente, o comportamento de uma variável quando outra variável do sistema, ou ela mesma, sofre um choque (impulso) naquele determinado instante $t$, que se transfere para o período futuro, em $t+1, t+2$, e assim por diante. Desse modo, é possível prever antecipadamente o que acontece com cada variável diante de um choque em qualquer variável do modelo, por meio da estrutura de defasagens e de análise conjunta efetuada pelo método.

A forma matemática do modelo VAR é a seguinte;

$$
y_{t}=A_{1} y_{t-1}+\ldots .+A_{N} y_{t-N}+B x_{t}+\varepsilon_{t}
$$

Onde:

. $y_{t}=$ vetor de variável endógena

- $x_{t}=$ vetor de variável exógena

- $A_{1+\cdots \ldots+} A_{N}$ e $B=$ matrizes dos coeficientes a serem estimados

. ${ }_{t}=$ vetor de inovações autocorrelacionado 
De acordo com a equação 1, é possível notar que as variáveis defasadas são as variáveis explicativas do sistema. Ao mesmo tempo em que as variáveis, no período $t$, são dependentes, verifica-se que, em $t-l$, as variáveis assumem a característica de independência. Essa é a principal característica de um modelo VAR, ou seja, as variáveis do sistema explicadas, também, pelo próprio passado $^{(4)}$. Um dos componentes mais importantes, do ponto de vista de previsão de uma variável em um modelo VAR, constitui-se na função de resposta a impulso, representada matematicamente por $\left(\varepsilon_{t}\right)$ na equação (1), que é o vetor de inovações autocorrelacionado. A função de resposta a impulso tem sido a peça central em análises econométricas que se utilizam de modelos VAR, demonstrando o comportamento futuro de uma variável no sistema estimado após um choque ${ }^{(5)}$.

Para os propósitos deste estudo, foi estimado um modelo VAR com dados de periodicidade mensal. Com isso foram evitadas suposições arbitrárias quanto aos valores dos preços das ações em dias faustos, como no estudo de Tabak e Lima (2003), cuja decisão foi pela inclusão de dados ausentes por interpolação cúbica, ou, ainda, Lamounier e Nogueira (2007), que repetiram o índice do dia anterior em feriados nacionais. A amplitude do período, de doze anos, e o número de observações, superior a cem, asseguram a estimativa com periodicidade mensal. Cumpre ressaltar que o período inicial das observações é janeiro de 1999, justamente o mês em que ocorreu a mudança do regime cambial brasileiro; e o período final, dezembro de 2010, decorreu da disponibilidade dos dados no período de realização da pesquisa. Aduz-se que o período selecionado contemplou os efeitos da crise financeira internacional, a qual teve origem no mercado de crédito dos Estados Unidos e impactou diretamente sobre os mercados acionários e preços das commodities.

Nesse sentido, as variáveis aplicadas na estimativa efetuada no pacote econométrico Eviews 6.0 são descritas, a seguir, com o objetivo de justificar os testes aplicados. Os gráficos com as variáveis podem ser visualizados na Figura 1, apresentada na quarta seção. Destaca-se, portanto, uma breve descrição de cada uma das séries utilizadas na estimativa, bem como a respectiva fonte:

1. Taxa de Câmbio: corresponde à taxa de câmbio nominal expressa pelo método europeu, ou seja, R \$/US\$, fim de período, extraída da base de dados Ipeadata (2006).

2. Preço de commodities: para avaliar a variação nos preços das commodities foi utilizado o Reuters/Jefferies CRB Index, um número índice que afere a variação dos preços de dezenove commodities como alumínio, cacau, café, petróleo, gás natural, ouro e prata, entre outras. $\mathrm{O}$ tradicional índice de commodities, atualmente calculado pela consultoria Jefferies em conjunto com a empresa de informações Reuters, surgiu em 1957 e possui credibilidade e transparência tanto na metodologia quanto na divulgação das informações. Os dados são disponibilizados pelo Commodity Research Bureau (n.d.).

3. Dow Jones: representa um dos principais índices da bolsa de valores dos EUA. Criado em 1896, o Dow Jones Industrial Average reflete a variação das ações de 30 empresas do setor industrial e o índice constitui uma referência em termos de tendências da economia estadunidense e dos mercados financeiros. A série foi extraída da base Economática (n.d.).

4. Ibovespa: é o principal índice de ações brasileiro, criado em 1968, a partir de uma carteira teórica de ações que, atualmente, corresponde a mais de $80 \%$ do número de negócios realizados no mercado à vista. Considerando os objetivos da pesquisa, foi utilizada a série do Ibovespa em dólares, extraída de Economática (n.d.).

5. Dummy sazonal: variável incluída com o objetivo de captar duas mudanças significativas no modelo VAR. A primeira mudança compreendeu o período de junho de 2002 a maio de 2003 e se justifica pelas incertezas decorrentes do cenário eleitoral brasileiro e da condução da política econômica pelo novo governo, que interferiu principalmente no comportamento da taxa de câmbio. A segunda mudança, que impactou diretamente o comportamento do índice Dow Jones, contemplou o período de abril de 2007 a fevereiro de 2009 e é explicado pelo agravamento da crise nos EUA, com o início do período marcado pelo pedido de proteção contra a falência por uma das maiores empresas de crédito imobiliário até a propagação dos efeitos da crise para a economia 
brasileira e para o mercado de commodities. Nesse sentido, a variável assumiu valor um nos dois períodos mencionados e valor zero no restante da amostra.

\section{Procedimentos de modelagem VAR}

Em vários estudos, modelos VAR foram estimados com poucas restrições sobre os parâmetros e, consequentemente, sobre o modelo. $\mathrm{O}$ desenvolvimento de novas técnicas permite selecionar o modelo mais adequado, confiável e estável. A seleção tem início na análise das séries temporais das variáveis, que podem ser estudadas em nível ou em diferenças, conforme os resultados dos testes de estacionariedade. A seleção também ocorre em termos de defasagens, com vários critérios disponíveis para o número de defasagens das variáveis explicativas. Pode-se ainda escolher entre um modelo com ou sem correção de erros. Finalmente, em função da forma da decomposição escolhida, a ordenação das variáveis constitui estratégia importante na modelagem, conforme será demonstrado adiante.

Para as variáveis utilizadas na estimativa aplicou-se o teste de raiz unitária Dickey Fuller e/ou Dickey Fuller aumentado, conforme Dickey e Fuller (1979). O número de defasagens foi selecionado de acordo com o melhor critério de Schwarz e, adicionalmente, com a escolha do número de defasagens necessária para remover qualquer tipo de correlação serial dos resíduos. Assim, apenas as séries diferenciadas foram consideradas estacionárias. Os resultados do teste podem ser observados na Tabela 1 e o comportamento das variáveis diferenciadas na Figura 1, ambas apresentadas na próxima seção. Vale ressaltar que foi efetuada uma transformação logarítmica nas variáveis com o objetivo de analisar a elasticidade entre os dados do modelo estimado nas funções de resposta a impulso. Solucionada a questão da não estacionariedade das séries, procedeu-se a escolha do melhor modelo multivariado em termos de número de defasagens. O aumento ou redução no número de defasagens das variáveis independentes pode acarretar instabilidade no modelo e perda de poder preditivo. Nesse sentido, foram aplicados cinco testes/critérios para a seleção do melhor modelo: Teste estatístico LR sequencial modificado com cada erro ao nível de 5\% de significância, Teste do erro de predição final, Critério de Akaike, Critério de Schwarz e Critério de Hannan-Quinn. Os testes são discutidos de forma detalhada por Lütkepohl (1991). Embora seja possível selecionar o melhor número de defasagens no intervalo [0,n], optou-se pela seleção no intervalo [1,5]. A opção no intervalo [1,5] é plenamente justificável, pois os efeitos dinâmicos do modelo VAR tornam-se limitados com uma estimativa sem defasagem. Os resultados indicaram a seleção de um modelo VAR(1) como o mais adequado de acordo com a totalidade dos testes. Assim, as equações do modelo estimado, com dados mensais, apresentaram o seguinte formato:

$$
\begin{aligned}
& T c_{t}=\alpha_{1}+\beta_{0} T c_{t-1}+\beta_{1} P c_{t-1}+\beta_{2} D j_{t-1}+\beta_{3} I b v_{t-1+} \beta_{4} D s_{t-1} \\
& P c_{t}=\alpha_{2}+\beta_{5} T c_{t-1}+\beta_{6} P c_{t-1}+\beta_{7} D j_{t-1}+\beta_{8} I b v_{t-1+} \beta_{9} D s_{t-1} \\
& D j_{t}=\alpha_{3}+\beta_{10} T c_{t-1}+\beta_{11} P c_{t-1}+\beta_{12} D j_{t-1}+\beta_{13} I b v_{t-1+} \beta_{14} D s_{t-1} \\
& I b v_{t}=\alpha_{4}+\beta_{15} T c_{t-1}+\beta_{16} P c_{t-1}+\beta_{17} D j_{t-1}+\beta_{18} I b v_{t-1+} \beta_{19} D s_{t-1}
\end{aligned}
$$

Em que:

. $\alpha_{1} \ldots \alpha_{4} \rightarrow$ constantes

. $\beta_{0} \ldots . . \beta_{19} \rightarrow$ parâmetros

. Tc $\rightarrow$ diferença do logaritmo natural da taxa de câmbio (R $\$ / U S \$)$

- $\mathrm{Pc} \rightarrow$ diferença do logaritmo natural do índice de preço das commodities

. $\mathrm{Dj} \rightarrow$ diferença do logaritmo natural do índice Dow Jones

. Ibv $\rightarrow$ diferença do logaritmo natural do índice Ibovespa em dólares 


\section{Ds $\rightarrow$ dummy sazonal}

De forma complementar, ressalta-se que, em séries não estacionárias, Granger e Newbold (1974) identificaram o problema da regressão espúria. Nesse contexto, o modelo VAR não é isento de eventual necessidade de um termo de correção de erros, que promove o ajustamento do comportamento a curto prazo entre as variáveis de acordo com o comportamento a longo prazo. Desse modo, torna-se importante testar a relação entre as equações do modelo para identificar possíveis vetores de cointegração. De uma forma geral, utiliza-se o teste de cointegração de Johansen com o objetivo de identificar os vetores de cointegração e estimar um modelo VEC ao invés de um VAR. A análise de cointegração constitui fator determinante na solução de problemas que envolvem relações entre séries não estacionárias.

Assim, aplicou-se o teste de cointegração de Johansen sobre as séries em nível, uma vez que foi detectada a presença de raiz unitária. A aplicação do teste de cointegração para as séries em nível com a presença de raiz unitária buscou identificar um ou mais vetores de cointegração entre as bolsas, preços das commodities e a taxa de câmbio. Foram utilizadas duas estatísticas: a estatística do traço e a do máximo autovalor. A execução do teste, conforme proposto por Johansen (1991), testa a hipótese nula de não cointegração versus a hipótese alternativa, indicando a quantidade de vetores de cointegração existentes no sistema. Conforme os dados apresentados na Tabela 3, na próxima seção, é possível perceber que não há indícios de vetores de cointegração, tanto pela estatística do traço quanto pela estatística do máximo autovalor.

A estabilidade do modelo VAR pode ser testada pela análise das raízes inversas do polinômio característico autorregressivo. Desse modo, para cada sistema multivariado, tem-se $k x p$ raízes, em que $k$ é o número de variáveis endógenas e $p$ representa a última defasagem utilizada no modelo. A análise das raízes demonstra se o modelo pode apresentar uma trajetória explosiva ou convergente. Tal análise foi realizada no presente estudo e constatou-se que as raízes inversas do polinômio característico autorregressivo encontram-se dentro do círculo unitário, o que assegura a estabilidade do modelo VAR.

Uma característica interessante do modelo VAR diz respeito à decomposição utilizada na geração do vetor de inovações autocorrelacionado e ao processo de ordenação das variáveis. Entre as várias decomposições possíveis, optou-se pela decomposição de Cholesky, em decorrência de sua ampla utilização pela literatura e dos efeitos dinâmicos proporcionados pelo método de ortogonalização das variáveis ${ }^{(6)}$. A decomposição de Cholesky é utilizada para a solução de sistemas lineares ( $\mathrm{n} \times \mathrm{n}$ ), cuja matriz do sistema seja simétrica e definida positiva. $\mathrm{O}$ cálculo matricial aplicado resulta na matriz diagonal de covariância das variáveis. Devido a esse procedimento utilizado na estimação, que atribui todo o efeito sistêmico à primeira variável do modelo, as alterações na ordem das variáveis, quando da estimação de um modelo VAR, podem ocasionar mudanças na função de resposta a impulso. Os procedimentos técnicos com relação a esse método podem ser verificados em Hamilton (1994).

Em função da atribuição do efeito sistêmico à primeira variável utilizada na estimativa, recomenda-se ordenar de acordo com o grau de endogeneidade, pois a ordenação pode resultar em funções de resposta a impulsos distintas para o mesmo conjunto de variáveis. As variáveis com maior poder de causalidade devem ser inseridas no início da sequência e as variáveis com menor poder de causalidade no final da sequência. Assim, com o objetivo de ordenar as variáveis de acordo com o grau de endogeneidade, foi utilizado o teste VAR Granger Causality/Block Exogeneity. Para cada equação do modelo VAR, o cálculo da estatística Wald testou a significância de cada uma das outras variáveis endógenas defasadas na equação. $\mathrm{O}$ valor total da estatística da variável demonstrou a significância de todas as outras variáveis endógenas na equação. Desse modo, a série que apresentou o menor valor da estatística referiu-se à variável com endogeneidade fraca e a que apresentou o maior valor pode ser caracterizada por endogeneidade forte. O teste permitiu, portanto, classificar as variáveis e gerar funções de resposta a impulso sob um critério estatisticamente consistente. Assim, os resultados do teste permitiram ordenar as variáveis de acordo com o grau de endogeneidade, conforme a Tabela 4, apresentada na próxima seção. 
Outro cuidado adotado na estimativa refere-se à necessidade de distribuição normal dos erros de previsão, um dos pressupostos de uma estimativa por mínimos quadrados ordinários. Embora alguns estudos descartem as imposições de testes e restrições sobre o modelo VAR, foi aplicado um teste de normalidade Jarque-Bera específico, que compara o terceiro e o quarto momento da distribuição dos resíduos com a distribuição normal, em uma análise multivariada. A execução do teste exige também a fatorização dos resíduos. Para tanto, aplicou-se o método da ortogonalização da covariância de acordo com Lütkepohl (1991), em que a matriz fatorizada é a inversa do fator triangular inferior de Cholesky da matriz de covariância dos resíduos. Testou-se, assim, a hipótese $\mathrm{H}_{0}$ de que os resíduos são normalmente distribuídos e se verificou que não ocorreu a normalidade dos erros, o que já era esperado em decorrência da característica de volatilidade de séries financeiras e macroeconômicas. Há que se ressaltar que a rejeição do teste não impede a interpretação e análise dos resultados, apesar de sugerir cautela ${ }^{(7)}$.

Além do teste global do modelo VAR, é possível testar a estabilidade dos parâmetros em cada uma das equações do modelo. Para tanto, utilizou-se o teste dos resíduos recursivos no sentido de avaliar eventuais instabilidades. $\mathrm{O}$ teste pode ser utilizado para dois propósitos: avaliar a estabilidade dos parâmetros e detectar quebras estruturais. Como a escolha do período já evitou uma quebra estrutural importante, que foi a mudança do regime cambial, foi possível identificar, em algumas equações, instabilidade intermitente, decorrente de oscilações bruscas nas variáveis. Vale salientar que eventual instabilidade pontual dos parâmetros não impede a interpretação dos resultados, de acordo com Bagliano e Favero (1998). Os resultados do teste dos resíduos recursivos, representados na Figura 2, podem ser observados na quarta seção.

Tradicionalmente, os efeitos de choques de um modelo VAR são apresentados em gráficos com as funções de resposta a impulso. É justamente a função de resposta a impulso que permite a visualização do efeito contágio. Assim, no eixo horizontal, optou-se por visualizar o movimento decorrente de choques até o sexto mês, visto que até esse período os choques dissiparam-se completamente. Para auxiliar na análise, as funções de resposta a impulso foram apresentadas considerando um choque hipotético de um desvio padrão em cada uma das variáveis, com as respectivas bandas de erro padrão geradas pelo método de Monte Carlo, com 1.000 repetições. Vale enfatizar que os movimentos das variáveis após um choque devem ser interpretados como elasticidades entre elas, em função da logaritmização das variáveis do sistema. As funções de resposta a impulso foram geradas a partir da decomposição de Cholesky, conforme mencionado anteriormente, que utiliza a inversa do fator de Cholesky da matriz de covariância dos resíduos para ortogonalizar os impulsos. O ordenamento das variáveis na estimativa foi realizado com base nos resultados do teste VAR Granger Causality/Block Exogeneity. Na derivação da matriz de Cholesky, foi aplicada, ainda, a correção dos graus de liberdade da matriz de covariância dos resíduos.

Adicionalmente, utilizou-se, como recurso adicional para suporte à hipótese deste estudo, a decomposição da variância dos erros de previsão. A decomposição da variância dos erros de previsão constitui instrumento adicional de análise e interpretação dos resultados. Resumidamente, consiste na identificação da responsabilidade de cada uma das variáveis na explicação da variância de todas as variáveis do sistema após um choque. De outra forma, pode-se afirmar que a decomposição da variância classifica a importância relativa de cada variável na determinação da própria variável e das demais. A metodologia de cálculo da decomposição de variância também se baseia na decomposição de Cholesky, o que implica reproduzir o mesmo raciocínio apresentado na descrição das funções de resposta a impulso, ou seja, mudanças na ordenação das variáveis implicam mudanças nos resultados da decomposição da variância. Por esse motivo, será utilizada a ordenação sugerida pelo teste VAR Granger Causality/Block Exogeneity. Para os propósitos deste estudo, calculou-se a decomposição da variância para um período de 24 meses após um choque aleatório, sendo apresentados os valores dos meses 1, 6, 12 e 24. Geralmente, nota-se que, a partir do $24^{\circ}$ mês, a participação estabiliza-se na maior parte dos modelos VAR. Em modelos estimados com uma ou mais séries em primeira diferença, a estabilização ocorre de forma mais acelerada e uma característica que também merece destaque é o fato de o componente autorregressivo da variável ser responsável por parcela importante da explicação 
desta. Mesmo assim, a decomposição da variância constitui importante mecanismo de análise em modelos multivariados.

\section{Resultados e Discussão}

A aplicação do teste de raiz unitária Dickey-Fuller para as variáveis selecionadas demonstrou a necessidade de se estimar um modelo com todas as séries diferenciadas. Conforme pode ser visualizado na Tabela 1, para as quatro séries em nível foi possível aceitar a hipótese nula de presença de raiz unitária.

Tabela 1

Teste de Raiz UnitáriaDickey-Fuller Aumentado (ADF)

\begin{tabular}{|c|c|c|c|c|c|c|c|}
\hline Variável & Defasagens & Constante & Tendência & $\mathrm{ADF}$ & $\begin{array}{c}\text { Valor Crítico } \\
10 \% \\
\end{array}$ & $\begin{array}{c}\text { Valor Crítico } \\
5 \% \\
\end{array}$ & $\begin{array}{c}\text { Valor Crítico } \\
1 \% \\
\end{array}$ \\
\hline Câmbio & 2 & $\operatorname{sim}$ & não & $-1.799885^{* * * *}$ & -2.577747 & -2.881978 & -3.477144 \\
\hline Commodities & 3 & $\operatorname{sim}$ & $\operatorname{sim}$ & $-2.596625 * * * *$ & -3.145608 & -3.442006 & -4.024452 \\
\hline Dow Jones & 0 & $\operatorname{sim}$ & não & $-2.059454 * * * *$ & -2.577591 & -2.881685 & -3.476472 \\
\hline Ibovespa & 1 & $\operatorname{sim}$ & $\operatorname{sim}$ & $-2.240685^{* * * *}$ & -3.145474 & -3.441777 & -4.023975 \\
\hline$\nabla$ Câmbio & 1 & não & não & $-6.911470^{*}$ & -1.615210 & -1.943107 & -2.581466 \\
\hline$\nabla$ Commodities & 1 & não & não & $-5.922948^{*}$ & -1.615210 & -1.943107 & -2.581466 \\
\hline$\nabla$ Dow Jones & 0 & não & não & $-11.20864 *$ & -1.615220 & -1.943090 & -2.581349 \\
\hline$\nabla$ Ibovespa & 0 & não & não & $-9.369985^{*}$ & -1.615220 & -1.943090 & -2.581349 \\
\hline
\end{tabular}

Nota. Fonte: Elaboração própria com base nos cálculos efetuados no pacote Eviews. (2007). (Versão 6.0) [Software] IrvineCalifórnia, USA: (QMS) Quantitative Micro Software.

*significativo em nível de 1\%, **significativo em nível de 5\%, ***significativo em nível de 10\%, **** aceitação da hipótese nula de presença de raiz unitária. Valores críticos gerados pelo pacote econométrico citado.

Em contrapartida, quando as séries foram diferenciadas, a hipótese nula pôde ser rejeitada em nível de significância de $1 \%$ para todas as séries. É possível observar a plotagem das séries em nível e diferenciadas na Figura 1. 

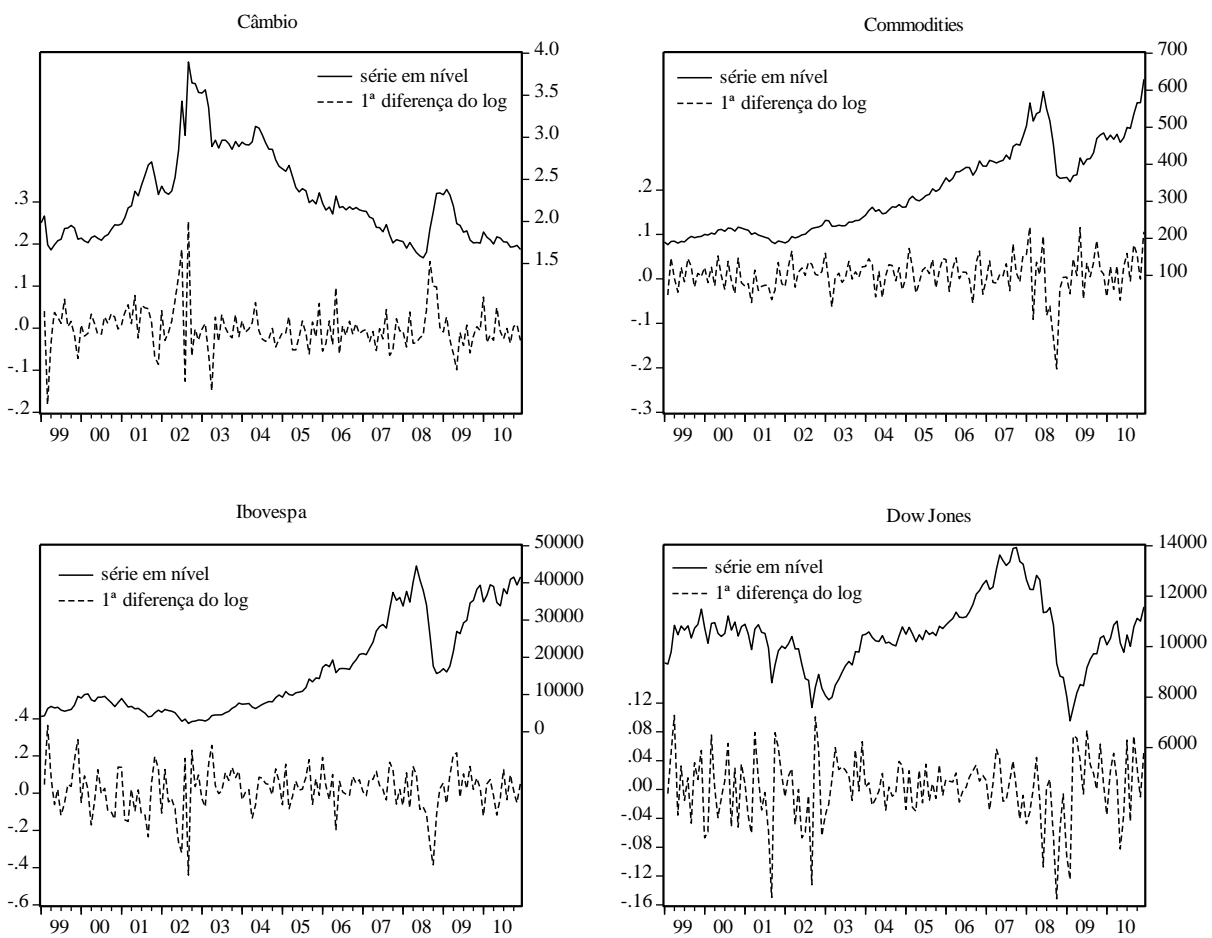

Figura 1. Variáveis em Nível e em Primeira Diferença.

Fonte: Economática - Tools for Investment Analysis (n.d.). Base de dados [CD-ROM]. São Paulo: Autor, Ipeadata. (2006). Base de dados [online]. Rio de Janeiro: IPEA. Recuperado de http://www.ipeadata.gov.br e Commodity Research Bureau. (n.d.). Reuters/Jefferies-CRB total return index. Recuperado de http://www.crbtrader.com/crbindex/crb_tr.xlsx

No que diz respeito ao número de defasagens do modelo, quatro dos cinco critérios utilizados apontaram o modelo VAR (1) como o melhor modelo, conforme demonstra a Tabela 2. Portanto o modelo será estimado com todas as variáveis em primeira diferença, com uma defasagem na relação de dependência, à exceção da variável dummy, que foi incluída em nível.

Tabela 2

\section{Seleção do Número de Defasagens do Modelo}

\begin{tabular}{llccccc}
\hline Ordem Var & Log likelihood & LR** & EPF*** & Akaike & Schwarz & Hannan-Quinn \\
\hline 1 defasagem & 989.4630 & 270.0934 & $6.29 \mathrm{e}-13^{*}$ & $-13.90526^{*}$ & $-13.26890^{*}$ & $-13.64666^{*}$ \\
2 defasagens & 1011.807 & 41.12603 & $6.55 \mathrm{e}-13$ & -13.86677 & -12.70011 & -13.39267 \\
3 defasagens & 1034.355 & $39.86709^{*}$ & $6.81 \mathrm{e}-13$ & -13.83123 & -12.13427 & -13.14163 \\
4 defasagens & 1048.125 & 23.34851 & $8.07 \mathrm{e}-13$ & -13.66847 & -11.44121 & -12.76337 \\
5 defasagens & 1057.079 & 14.53556 & $1.03 \mathrm{e}-12$ & -13.43593 & -10.67838 & -12.31533 \\
\hline
\end{tabular}

Nota. Fonte: Elaboração própria com base nos cálculos efetuados no pacote Eviews. (2007). (Versão 6.0) [Software] IrvineCalifórnia, USA: (QMS) Quantitative Micro Software.

* Seleção de acordo com o critério. **Teste estatístico LR sequencial modificado (cada teste ao nível de 5\%). ***Erro de predição final.

A hipótese de que os mercados financeiros apresentam relação de cointegração ou, ainda, de que há uma relação a longo prazo entre mercados acionários, preços de commodities e taxa de câmbio pode ser rejeitada com 5\% de significância, conforme os resultados do teste de cointegração de Johansen para as séries em nível. É possível perceber que não foram identificadas, portanto, relações a 
longo prazo entre o Índice Dow Jones, preços das commodities, taxa de câmbio e o Ibovespa, conforme reporta a Tabela 3 .

Tabela 3

Resultados do Teste de Cointegração de Johansen

\begin{tabular}{cccccccc}
\hline $\begin{array}{c}\text { Equações } \\
\text { hipotetizadas }\end{array}$ & Autovalor & $\begin{array}{c}\text { Estatística do } \\
\text { Traço }\end{array}$ & $\begin{array}{c}\text { Valor crítico } \\
\mathbf{5 \%}\end{array}$ & Prob. & $\begin{array}{c}\text { Estatística do } \\
\text { máx. autovalor }\end{array}$ & $\begin{array}{c}\text { Valor } \\
\text { crítico5\% }\end{array}$ & Prob. \\
\hline Nenhuma* & 0.176505 & 52.66132 & 69.81889 & $0.52^{* *}$ & 27.57605 & 33.87 & $0.23^{* *}$ \\
Até 1* & 0.096792 & 25.08527 & 47.85613 & $0.92^{* *}$ & 14.45590 & 27.58 & $0.79 * *$ \\
Até $2^{*}$ & 0.044411 & 10.62937 & 29.79707 & $0.97 * *$ & 6.450758 & 21.13 & $0.97 * *$ \\
Até 3* & 0.028490 & 4.178614 & 15.49471 & $0.89 * *$ & 4.104383 & 14.26 & $0.85^{* *}$ \\
Até 4* & 0.000523 & 0.074231 & 3.841466 & $0.79 * *$ & 0.074231 & 3.84 & $0.79 * *$ \\
\hline
\end{tabular}

Nota. Fonte: Elaboração própria com base nos cálculos efetuados no pacote Eviews. (2007). (Versão 6.0) [Software] IrvineCalifórnia, USA: (QMS) Quantitative Micro Software.

*indica rejeição da hipótese em nível de 5\% de significância. **Conforme valores extraídos de Mackinnon, J. G., Haug, A. A., \& Michelis, L. (1999). Numerical distribution functions of likelihood ratio tests for cointegration. Journal of Applied Econometrics, 14(5), 563-577. doi: 10.1002/(SICI)1099-1255(199909/10).

Já o teste de exogeneidade das variáveis indicou, conforme mostra a Tabela 4, que a variável com endogeneidade fraca representou os preços das commodities, além da variável dummy. Em contrapartida, a taxa de câmbio mostrou endogeneidade forte. Assim, a ordenação utilizada na decomposição de Cholesky foi a seguinte: dummy, commodities, Ibovespa, Dow Jones e câmbio.

Tabela 4

Teste de Exogeneidadedas Variáveis - VAR Granger Causality/Block ExogeneityWald Tests

\begin{tabular}{lcccccccccc}
\hline & \multicolumn{2}{c}{ Dummy } & \multicolumn{2}{c}{ Commodities } & \multicolumn{2}{c}{ Ibovespa } & \multicolumn{2}{c}{ Dow Jones } & \multicolumn{2}{c}{ Câmbio } \\
\cline { 2 - 11 } & $\chi^{2}$ & Prob. & $\chi^{2}$ & Prob. & $\chi^{2}$ & Prob. & $\chi^{2}$ & Prob. & $\chi^{2}$ & Prob. \\
\hline Dummy & & & 1.20 & 0.27 & 1.53 & 0.22 & 8.14 & 0.01 & 0.62 & 0.43 \\
Commodities & 0.06 & 0.80 & & & 0.01 & 0.93 & 0.28 & 0.60 & 0.00 & 0.95 \\
Ibovespa & 0.95 & 0.33 & 0.83 & 0.36 & & & 0.65 & 0.42 & 10.92 & 0.00 \\
Dow Jones & 2.72 & 0.10 & 0.03 & 0.87 & 0.60 & 0.44 & & & 0.69 & 0.41 \\
Câmbio & 0.06 & 0.81 & 0.22 & 0.64 & 7.57 & 0.01 & 0.00 & 0.97 & & \\
\hline Total & 2.87 & 0.58 & 2.90 & 0.57 & 8.97 & 0.06 & 9.39 & 0.05 & 13.60 & 0.01 \\
\hline
\end{tabular}

Nota. Fonte: Elaboração própria com base nos cálculos efetuados no pacote Eviews. (2007). (Versão 6.0) [Software] IrvineCalifórnia, USA: (QMS) Quantitative Micro Software.

Também, foi aplicado o teste Jarque-Bera com o objetivo de se avaliar a normalidade dos resíduos do modelo. Apesar de a hipótese de normalidade não se confirmar, o modelo foi estimado mesmo com a ocorrência de nãonormalidade dos resíduos, sem comprometimento dos resultados, conforme discutido anteriormente. 

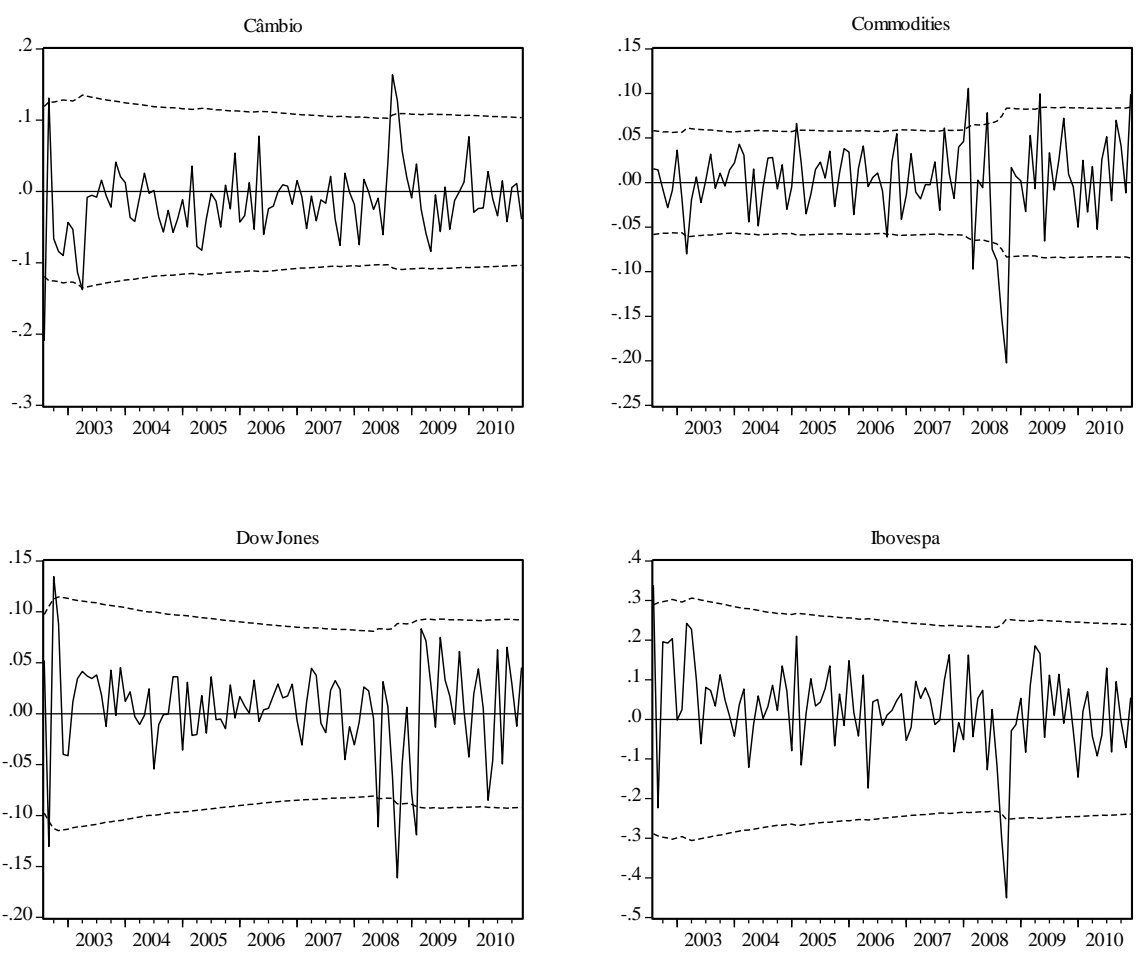

Figura 2. Teste de Estabilidade dos Parâmetros - Resíduos Recursivos.

Fonte: Pacote Eviews. (2007). (Versão 6.0) [Software] Irvine-Califórnia, USA: (QMS) Quantitative Micro Software.

Assim, considerando a hipótese de nãonormalidade da distribuição residual, a Figura 2 apresenta o teste de resíduos recursivos, utilizado com o objetivo de avaliar a estabilidade dos parâmetros. Verificou-se, então, instabilidade presente nos parâmetros de todas as equações em 2008, em decorrência da crise financeira internacional, inclusive na equação do Dow Jones. De uma forma geral, as equações estimadas apresentaram parâmetros estáveis em praticamente todo o período, à exceção da equação das commodities, com instabilidade em vários períodos da amostra; e, no início da amostra, nos casos dos índices Dow Jones e Ibovespa, além da taxa de câmbio. 
Response to Cholesky One S.D. Innovations \pm 2 S.E.
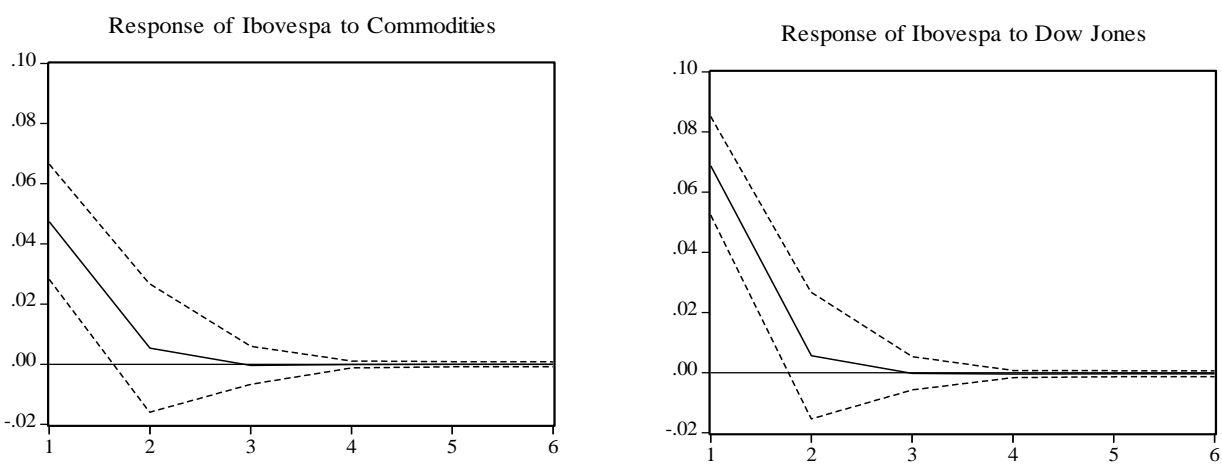

Response of Ibovespa to Câmbio

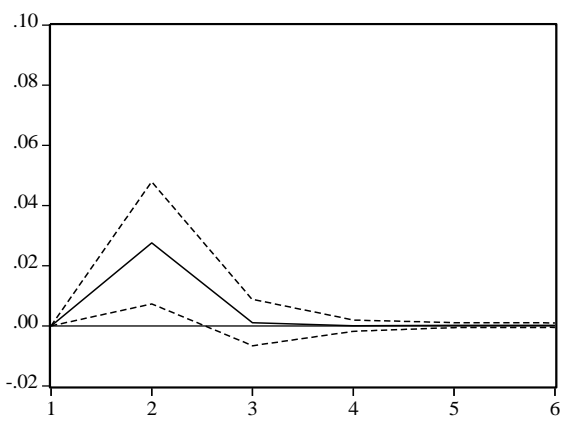

Figura 3. Funções de Resposta do Ibovespa a Impulsos.

Fonte: Pacote Eviews. (2007). (Versão 6.0) [Software] Irvine-Califórnia, USA: (QMS) Quantitative Micro Software.

Após a análise das funções de resposta a impulsos, cabe verificar a participação relativa de cada uma das variáveis por meio da decomposição da variância dos erros de previsão. Desse modo, a Tabela 5indica que, no primeiro mês após o choque, o Ibovespa é explicado, pelo índice Dow Jones e pelo comportamento das commodities, em $33,10 \%$ e $15,71 \%$, respectivamente.

Tabela 5

Decomposição da Variância dos Erros de Previsão do Ibovespa

\begin{tabular}{ccccc}
\hline Mês & Commodities & Dow Jones & Ibovespa & Câmbio \\
\hline $\mathbf{1}$ & 15.71091 & 33.09683 & 50.17233 & 0.000000 \\
$\mathbf{6}$ & 14.81129 & 31.01399 & 47.65513 & 4.955359 \\
$\mathbf{1 2}$ & 14.75844 & 30.90624 & 47.48922 & 4.939652 \\
$\mathbf{2 4}$ & 14.73064 & 30.84956 & 47.40195 & 4.929863 \\
\hline
\end{tabular}

Nota. Fonte: Elaboração própria com base nos cálculos efetuados no pacote Eviews. (2007). (Versão 6.0) [Software] IrvineCalifórnia, USA: (QMS) Quantitative Micro Software.

Já a taxa de câmbio não aparece como responsável pelo comportamento do Ibovespa no primeiro mês após o choque, mas, no sexto mês, assume a parcela de responsabilidade de 4,96\% do comportamento do índice, substituindo de forma parcial a participação do índice Dow Jones na explicação, que se reduz para $31,01 \%$; e do próprio Ibovespa, que passa a ser de 47,66\%. A variável dummy apresenta-se com aproximadamente $1 \%$ de explicação do comportamento do índice no primeiro mês e, mesmo após 24 meses, mantém-se abaixo de $2,5 \%$, motivo pelo qual foi suprimida da tabela. $\mathrm{O}$ fraco poder explicativo da variável dummy explica-se pelo fato de a relação dinâmica da bolsa com as demais variáveis analisadas no presente estudo ser independente da crise. Em outras palavras, ainda que a crise tenha provocado uma mudança de tendência na trajetória da bolsa, o efeito foi pontual e 
não alterou a dinâmica presente entre as variáveis. Mesmo com a crise, o comportamento a curto prazo do Ibovespa continuou dependente de seu passado e do comportamento do índice Dow Jones.

\section{Considerações Finais}

O presente artigo teve o objetivo de analisar o efeito contágio do índice Dow Jones e dos preços das commodities sobre o Ibovespa sob o comportamento do regime de câmbio flutuante, em vigor, no Brasil, desde 1999, além de avaliar eventual influência da taxa de câmbio sobre o índice de ações brasileiro. As relações foram analisadas sob a ótica teórica do efeito contágio por meio da estimativa de um modelo VAR, com as respectivas funções de resposta a impulso e decomposição da variância dos erros de previsão. Adicionalmente, foi realizada uma análise de cointegração, que detecta relacionamentos a longo prazo entre as variáveis, além de testes de causalidade e endogeneidade.

Conquanto a extensa literatura sobre o efeito contágio e cointegração entre bolsas americanas/europeias e bolsas de países emergentes, entre as quais, destaca-se a brasileira, percebe-se que análises efetuadas exclusivamente sob um regime de câmbio flutuante são menos recorrentes. Incomum também é a inclusão de variáveis nos modelos que representem preços de commodities, apesar da natureza das empresas e dos setores que predominam no índice de ações brasileiro, em sua maioria, fortemente concentrados em matérias-primas. Vale destacar ainda que a inclusão de preços de commodities e da taxa de câmbio permite uma análise apurada dos canais de ocorrência do efeito contágio.

Inicialmente, pôde-se constatar que não há evidências de cointegração entre as bolsas brasileira e americana. Também não há evidências de relações a longo prazo entre o Ibovespa, preço das commodities e taxa de câmbio. Especificamente em relação à cointegração de bolsas, a estimativa corroborou os resultados já encontrados pela literatura (que apresenta análises semelhantes para outros períodos) e refutou parte da hipótese desta pesquisa, ou seja, de que haveria uma relação a longo prazo entre o comportamento dos preços das ações das bolsas de valores sob um regime de câmbio flutuante.

A cointegração, no entanto, não é a única evidência de relação entre variáveis. Conforme pôde ser constatado, há evidências de relações importantes a curto prazo verificadas com a aplicação do teste de exogeneidade/causalidade, como a fraca endogeneidade do preço das commodities, conforme já era esperado em decorrência de sua formação de preços no mercado internacional e forte endogeneidade da taxa de câmbio. Pelo teste, foi possível constatar que ocorre o efeito contágio, visto que o comportamento dos preços das commodities causa o comportamento do Dow Jones e do Ibovespa. Já a taxa de câmbio foi resultante do comportamento da bolsa de valores brasileira, assim como das demais variáveis do sistema estimado.

A curto prazo, portanto, os retornos de aplicações financeiras em fundos de commodities afetam os retornos das aplicações no Ibovespa no mesmo sentido, eliminando a possibilidade de diversificação de portfólios e indicando a presença do efeito contágio. A longo prazo, entretanto, não há evidências de comportamento similar entre os dois mercados, o que justificaria eventual diversificação entre ações brasileiras e fundos de commodities. Outra questão que merece destaque é o comportamento instável das variáveis, em 2008, apontado pelo teste dos resíduos recursivos. Nas quatro equações, notou-se forte instabilidade no período, justificada pela crise que impactou os mercados financeiros internacional e nacional.

Já as funções de resposta a impulsos permitiram corroborar, parcialmente, a hipótese inicialmente formulada. Conforme verificado, o Ibovespa apresenta elasticidade positiva com o preço das commodities e com o índice Dow Jones. Em outras palavras, pode-se afirmar que um aumento no preço das commodities ou das ações de empresas norte-americanas influencia positivamente o índice brasileiro. Não obstante, a decomposição da variância dos erros de previsão destaca os preços das 
commodities e o comportamento do Dow Jones como os principais responsáveis pela trajetória da bolsa brasileira.

Em razão dos propósitos deste estudo, não foram avaliados eventuais impactos de variáveis econômicas de natureza exclusivamente interna, como o nível de produção, consumo ou taxa de juros do mercado monetário sobre o comportamento do Ibovespa. Considerando que os preços das commodities exercem um papel relevante sobre a trajetória do Ibovespa, destaca-se a necessidade de ampliar o modelo especificado com outras variáveis, além de aprofundar a discussão de causalidade do índice sobre a taxa de câmbio, detectada pelo teste de endogeneidade. De acordo com os resultados obtidos neste estudo e pela relevância apresentada, incluem-se tais questões como tópicos importantes de agenda para pesquisas futuras.

\section{Artigo recebido em 21.02.2012. Aprovado em 01.06.2012.}

\section{Notas}

\footnotetext{
${ }^{1}$ O presente artigo corresponde a uma versão aprimorada de trabalho apresentado no ENANPAD de 2010.
}

${ }^{2}$ A primeira estimativa, realizada por Sims (1980) envolveu a comparação de variáveis macroeconômicas entre as economias da Alemanha e EUA.

${ }^{3}$ Uma discussão extremamente crítica do modelo VAR pode ser observada no artigo de Cooley, T., \& Leroy, S. (1985). Atheoretical macroeconometrics: a critique. Journal of Monetary Economics, 16(3), 283-308. doi: 10.1016/03043932(85)90038-8

${ }^{4}$ É possível ainda estimar um modelo VAR com as variáveis independentes em $t$, embora tal procedimento seja pouco usual e com perda de informações importantes na geração das funções de resposta a impulso.

${ }^{5}$ A utilização da função de resposta a impulso pode ser observada em Pimenta (2004), Grôppo (2006) e Lamounier e Nogueira (2007).

${ }^{6}$ A decomposição de Cholesky foi utilizada, especificamente para análise de mercados financeiros, por Pimenta (2004) e Tabak e Lima (2003). Outras aplicações com vetores autorregressivos que utilizam a decomposição podem ser observadas em Minella, A. (2001). Monetary policy and inflation in Brazil (1975-2000): a VAR estimation. [WorkingPaper Series $n^{\circ}$ 33]. Banco Central do Brasil, Brasília, DF, Pinheiro, A., \& Amin, M. (2005, dezembro). Fluxos de capitais e componentes macroeconômicos: análise de inter-relações através da aplicação de um modelo de vetores auto-regressivos (VAR). Anais do Encontro Nacional de Economia, Natal, RN, Brasil, 33 e Oreiro, J., Paula, L., Silva, G., \& Ono, F. (2006). Determinantes macroeconômicos do spread bancário no Brasil: teoria e evidência recente. Economia Aplicada, 10(4), 609-634. doi: 10.1590/S1413-80502006000400007.

\footnotetext{
${ }^{7}$ A não normalidade dos resíduos em análises de séries macroeconômicas brasileiras é comum nos estudos que realizam o testeJarque-Bera, como, por exemplo, Minella (2001), Pinheiro e Amin (2005) e Oreiro et al. (2006). Especificamente em relação ao Ibovespa, Medeiros, O. T., \& Van Doornik, B. F. (2008). A relação empírica entre dividendos, volatilidade de retornos e volume de negócios no mercado de ações brasileiro. Brazilian Business Review, 5(1), 1-17 também não encontraram evidências de normalidade na distribuição dos erros do índice.
}

\section{Referências}

Bagliano, F., \& Favero, C. (1998). Measuring monetary policy with VAR models: an evaluation. European Economic Review, 42(6), 1069-1112. doi: 10.1016/S0014-2921(98)00005-1

Barr, G., \& Kantor, B. (2002). The South African economy and its asset markets: an integrated approach. South African Journal of Economics, 70(1), 53-77. doi: 10.1111/j.18136982.2002.tb00038.x

Caselani, C. N., \& Eid, W., Jr. (2005, julho). A influência dos determinantes microeconômicos e macroeconômicos sobre a volatilidade das ações negociadas no Brasil. Anais do Encontro Brasileiro de Finanças, São Paulo, SP, Brasil. 
Commodity Research Bureau. (n.d.). Reuters/Jefferies-CRB total return index. Recuperado de http://www.crbtrader.com/crbindex/crb_tr.xlsx

Dickey, D., \& Fuller, W. (1979). Distribution of the estimators for autoregressive time series with a unit root. Journal of the American Statistical Association, 74(366), 427-431. doi: $10.2307 / 2286348$

Dornbusch, R., Park, Y. C., \& Claessens, S. (2000). Contagion: understanding how it spreads. World Bank Research Observer, 15(2), 177-197. doi: 10.1093/wbro/15.2.177

Economática - Tools for Investment Analysis (n.d.). Base de dados [CD-ROM]. São Paulo: Autor.

Eviews. (2007). (Versão 6.0) [Software] Irvine-Califórnia, USA: (QMS) Quantitative Micro Software.

Forbes, K., \& Rigobon, R. (2002). No contagion, only interdependence: measuring stock market comovements. The Journal of Finance, 57(5), 2223-2261. doi: 10.1111/0022-1082.00494

Fifield, S. G. M., Power, D. M., \& Sinclair, C. D. (2002). Macroeconomic factors and share returns: an analysis using emerging market data. International Journal of Finance and Economics, 7(1), 51-62. doi: 10.1002/ijfe.173

Fundo Monetário Internacional. (2008). Exchange arrangements and foreign exchange markets: developments and issues. Recuperado de http://www.imf.org/external/np/mfd/er/2008/eng/0408.htm

Gamboa, U. M. (2006). Análise da sustentabilidade através da política fiscal brasileira através da história: um exercício de cliometria de Dom Pedro I a Lula (Tese de doutorado). Universidade de São Paulo, São Paulo, SP, Brasil.

Granger, C. W. J., \& Newbold, P. (1974). Spurious regressions in econometrics. Journal of Econometrics, 2(2), 111-120. doi:10.1016/0304-4076(74)90034-7

Grôppo, G. S. (2006). Relação dinâmica entre Ibovespa e variáveis de política monetária [Edição Especial]. Revista de Administração de Empresas, 46, 72-85.

Grôppo, G. S., Amaral, H. F., Bertucci, L. A., \& Barros, L. C. (2001, setembro). Integração de mercados: BOVESPA, MERVAL e DOW JONES. Anais do Encontro Nacional da Associação Nacional de Pós-Graduação e Pesquisa em Administração, Campinas, SP, Brasil, 25.

Hamilton, J. (1994). Time series analysis. Princeton: Princeton University Press.

Ipeadata. (2006). Base de dados [online]. Rio de Janeiro: IPEA. Recuperado de http://www.ipeadata.gov.br

Johansen, S. (1991). Estimation and hypothesis testing of cointegration vectors in gaussian vector autoregressive models. Econometrica, 59(6), 1551-1580.

Lamounier, W. M., \& Nogueira, E. M. (2007). Causalidade entre os retornos de mercados de capitais emergentes e desenvolvidos. Revista de Contabilidade e Finanças USP, 18(43), 34-48. doi: $10.1590 /$ S1519-70772007000100004

Leal, R. P. C., \& Costa, N. C., Jr. (1998). A integração entre as bolsas de valores de Buenos Aires e São Paulo. Revista de Administração Contemporânea, 2(1), 87-99. doi: 10.1590/S141565551998000100006

Lütkepohl, H. (1991). Introduction to multiple time series analysis. New York: Springer-Verlag. 
Mackinnon, J. G., Haug, A. A., \& Michelis, L. (1999). Numerical distribution functions of likelihood ratio tests for cointegration. Journal of Applied Econometrics, 14(5), 563-577. doi: 10.1002/(SICI)1099-1255(199909/10)

Nogueira, E. M., \& Lamounier, W. M. (2008). "Contágio" entre mercados de capitais emergentes e mercados desenvolvidos: evidências empíricas e reflexos sobre a diversificação internacional de portfólios. Revista Brasileira de Finanças, 6(2), 267-286.

Pereira, A. F. O. A., Dantas, A. B., \& Costa, N. C., Jr. (2002, setembro). Estimação da co-integração das principais bolsas da América Latina, Estados Unidos e Japão pela metodologia Johansen. Anais do Encontro Nacional da Associação Nacional de Pós-Graduação e Pesquisa em Administração, Salvador, BA, Brasil, 26.

Pericoli, M., \& Sbracia, M. (2003). A primer on financial contagion. Journal of Economic Surveys, 17(4), 571-608. doi: 10.1111/1467-6419.00205

Pimenta, T., Jr. (2004). Uma mensuração do fenômeno da interdependência entre os principais mercados acionários da América Latina e NASDAQ. Revista de Administração da USP, 39(2), $177-185$.

Sims, C. A. (1980). Macroeconomics and reality. Econometrica, 48(1), 1-48.

Sims, C. A. (1986). Are forecasting models usable for policy analysis? Federal Reserve Bank of Minneapolis Quarterly Review, 10(1), 2-16.

Sanvicente, A. Z. (1998). A integração do mercado brasileiro de ações ao mercado internacional: uma aplicação de análise de cointegração. Resenha BMF, 125, 31-43.

Tabak, B., \& Lima, E. (2003). Causality and cointegration in stock markets: the case of Latin America. Revista Brasileira de Economia de Empresas, 3(2), 27-45. 